\title{
Connecting DNA Origami Structures into a Designed and Functionalized Network
}

\author{
George Amoako ${ }^{1}$ \\ ${ }^{1}$ University of Cape Coast, Ghana \\ Correspondence: George Amoako, University of Cape Coast, Ghana. E-mail: gamoako@ucc.edu.gh
}

Received: July 31, 2019

Accepted: September 26, 2019 Online Published: September 30, 2019

doi:10.5539/jmsr.v8n4p14

URL: https://doi.org/10.5539/jmsr.v8n4p14

\begin{abstract}
The versatility of the DNA origami approach of organizing nanoparticles at the nanometer scale, together with thiol chemistry have been used. These approaches were used to design DNA origami structures and to functionalize them with gold nanoparticles after designing attachment sites on the DNA origami structures. In all two structures were designed - a cross-like structure and a nanotube but only the nanotube structure was used to form the gold nanoparticle helices. Finally, use was made of the specific affinity interaction between biotin and streptavidin to connect the DNA origami templated AuNP helices to the cross-like structure. Agarose gel electrophoresis, UVvis spectroscopy and TEM were used to characterize the structure.
\end{abstract}

Keywords: DNA Origami, Helices, Gel Electrophoresis, TEM, Thiol Chemistry, Sequences

\section{Introduction}

On the nanometer scale matter often behaves differently with respect to the bulk properties of the same material. This has initiated a wealth of research work to understand, characterize, describe and predict what happens at the nanoscale level (Moriarty, 2001; Rosei, 2004; Ratner \& Ratner, 2003). The approaches used to synthesize materials can be split into two main categories; top down approaches, where the processing begins with a bulk material from which a nanomaterial is fabricated, and bottom up approaches, in which the nanomaterial is built up from finer scales (Ramesh, 2009). There are many established synthetic routes to the production of nanoparticles of controlled morphology and composition, the programmed spatial arrangement of nanoparticles remains a challenge. Nanoparticles arrays in an effective medium with prescribed properties can be produced through AFM manipulation, e-beam lithography, focused ion beam milling, direct laser writing, nanoimprint lithography, interference optical lithography, etc (Cai \& Shalaev, 2010; Boltasseva \& Shalaev, 2008; Maier, 2001).

However, self-assembly provides an attractive alternative if appropriate methods can be developed which will allow control over size and geometry of designed objects. There are many strategies and approaches available for bioconjugation of nanoparticles, including attachment to biotin-avidin (Bruchez, Moronne, Gin, Weiss, \& Alivisatos, 1998; Connolly \& Fitzmaurice, 1999), antigen-antibodies (Shenton, Davis, \& Mann, 1999), peptides (Whaley, English, Hu, Barbara, \& Belcher, 2000; Hermanson, 1996), proteins (Hermanson, 1996), DNA (Storhoff \& Mirkin, 1999), etc.

The theoretical framework establishing the use of DNA as a structural building material to pursue the construction of devices on a few hundred nanometers scale was laid down by Seeman (Seeman, 1982; Seeman, 2003). This is possible due to the capacity for programmable self-assembly and the stability, stiffness, and flexibility exhibited by DNA. Due to enhanced automated methods and the usefulness of polymerase chain reaction (PCR) techniques that can amplify DNA sequences from microscopic to macroscopic quantities, DNA has become a powerful structural tool (Sun \& Kiang, 2005). With the self-assembling property of DNA well grounded, Rothemund (Rothemund, 2006) introduced the method of DNA origami which is versatile in constructing arbitrary designs. For multi-layer objects, the mixture is annealed over a certain temperature range (Rothemund, 2006; Douglas et al., 2009b; Castro et al., 2011) and several days are needed for folding (Douglas et al., 2009b; Castro et al., 2011; Douglas et al., 2009a).

With the development of these nanoscale synthesis approaches have come the formation of diverse nanoparticle structures. There is a great deal of potential including both plasmonic and electronic applications when DNA is used to structure metals at the nanoscale. The DNA origami approach developed by Rothemund (Rothemund, 
2006) has improved the addressability of using DNA as a template for organizing nanoparticles. The use of DNA origami as a template to assemble nanoparticles is due to the fact that the individual staples can be extended with additional sequences (sticky ends) which protrude on the surface. After acquiring the desired shape, the attachment sites are also designed. Each individual staple involved in binding nanoparticles to the DNA origami template can be modified and extended with sticky ends. Gold nanoparticles functionalized with complementary sequences can then hybridize with these sticky ends and attach themselves on the template by making use of the covalent bond between sulfur and gold (Nuzzo \& Allara, 1983; Dubois \& Nuzzo, 1992; Herne \& Tarlov, 1997; Yang, Yau, \& Chan, 1998; Bain \& Whitesides, 1989; Storhoff, Elghanian, Mucic, Mirkin, \& Letsinger, 1998; Jin, Wu, Li, Mirkin, $\&$ Schatz, 2003). This approach has been used extensively to organize metal nanostructures into several different shapes.

Recently, Pilo-Pais and coworkers (Pilo-Pais, Goldberg, Samano, LaBean, \& Finkelstein, 2011) used rectangular DNA origami to arrange gold nanoparticles into several shapes including rings, pairs of parallel bars, and $\mathrm{H}$ shapes which had outer dimensions that were close to that of the DNA origami. Ding et al. (Ding et al., 2010) also made use of triangular DNA origami templates to rationally arrange a self-similar linear chain of six metal nanospheres. The use of DNA origami templates to arrange chiral plasmonic structures have been demonstrated (Kuzyk, 2012; Shen et al., 2012). Shen and his co-authors (Shen et al., 2012) used a DNA origami template to design gold attachment sites and attached one gold nanoparticle to each of these sites. They then added DNA folding strands which rolled up the rectangular sheets into a hollow DNA tube resulting in the nanoparticles being arranged into a helical geometry. To show the helical arrangement of nanoparticles using DNA origami, Kuzyk and colleagues (Kuzyk, 2012) used the caDNAno honeycomb-pleated approach to design a hollow DNA origami tube as template. They designed Left- and Right- handed nanohelices that were formed by nine gold nanoparticles each of diameter $10 \mathrm{~nm}$. Acuna et al. (Acuna et al., 2012) used DNA origami structures as breadboard to position both fluorophore and metal nanoparticles with nanometer precision. They then performed single molecule fluorescence quenching studies of a fluorophore by metallic nanoparticles.

Due to the unique optical properties of metal nanoparticles, it will be of particular interest if they are structured with precise shapes and separation between them. When these precise parameters are achieved, plasmonic and CD (Kuzyk, 2012; Shen et al., 2012) characteristics of these metal nanostructures can be studied.

In this work, the strong biotin-streptavidin binding would be used to connect several origami objects that are functionalized with Au nanoparticles. These origami objects are well characterized in (Amoako et al., 2013; Amoako et al., 2015).

\section{Materials and Methods}

Materials, methods and folding of the DNA origami objects are well described and characterized in (Amoako et al., 2013; Amoako et al., 2015).

The procedures following were adapted (Castro et al., 2011; Zhao, Jacovetty, Liu, \& Yan, 2011; Tanton, 2002).

\subsection{Phosphination and Concentration of AuNPs}

The $10 \mathrm{~nm}$ AuNPs were stabilized with absorption of BSPP. BSPP (15 mg) was added to the colloidal AuNP solution $(50 \mathrm{~mL})$ and the mixture shaken overnight with a constant temperature incubator at room temperature. Sodium chloride (solid) was slowly added to the mixture and stirred until the color changed from deep burgundy to light purple. The resulting mixture was centrifuged at $3000 \mathrm{rpm}$ for $30 \mathrm{~min}$ and the supernatant was carefully removed with a pipette. AuNPs were resuspended in $1 \mathrm{~mL}$ solution of BSPP $(0.5 \mathrm{mM}) .500 \mu \mathrm{L}$ methanol was then added to precipitate the particles and the mixture was centrifuged at $3000 \mathrm{rpm}$ for $30 \mathrm{~min}$ again. The supernatant was carefully removed and AuNPs were resuspended in $1 \mathrm{~mL}$ of $0.5 \mathrm{mM}$ BSPP. The concentration of the AuNPs was estimated by the optical absorbance at $520 \mathrm{~nm}$. Phosphine coating increases the negative charge on the particle surface and therefore stabilizes the AuNPs in high electrolyte concentrations at high particle density.

\subsection{Preparation of AuNP-DNA Conjugates}

TCEP $(20 \mathrm{mM}, 1 \mathrm{~h})$ in water was used to reduce the disulfide bond in the thiol modified oligonucleotides to monothiol. Size exclusion columns (G-25, GE Healthcare) were used to purify the oligonucleotides and to remove smaller molecules. Monothiol modified oligonucleotides and phosphinated AuNPs were then combined (DNA to AuNP molar ratio of more than 200:1) in 0.5X TBE buffer ( $89 \mathrm{mM}$ Tris, $89 \mathrm{mM}$ boric acid, $2 \mathrm{mM}$ EDTA, pH 8.0) containing $50 \mathrm{mM} \mathrm{NaCl}$ for 40 hours at room temperature to ensure the AuNPs were fully covered by thiolated DNA. 0.5X TBE buffer in Microcon (100KDa, Millipore, Billerica, MA) columns were used to wash AuNP-DNA conjugates to remove the excess oligonucleotides. The concentration of these AuNP-DNA conjugates was estimated from the optical absorbance at $520 \mathrm{~nm}$. Freshly prepared AuNPs $(10 \mathrm{~nm})$, fully covered with DNA 
strands did not precipitate in buffer ( $5 \mathrm{mM}$ Tris, $1 \mathrm{mM}$ EDTA, $12 \mathrm{mM} \mathrm{MgCl}$ ), which is preferred for the folding in DNA origami. This high salt resistant property of fully covered AuNPs makes it possible to assemble them on the DNA origami template.

\subsection{Preparation and Purification of Origami-AuNPs Complexes}

The purified DNA origami was then mixed with AuNP-DNA conjugates in a ratio of 1:10 and annealed from 40 ${ }^{0} \mathrm{C}$ to room temperature. The annealed product of the DNA origami and AuNPs was loaded in a $1.0 \%$ Gel-red (Biotum) stained agarose gel (running buffer $0.5 \mathrm{X}$ TBE containing $11 \mathrm{mM} \mathrm{MgCl}_{2}$, loading buffer $50 \%$ glycerol, $70 \mathrm{~V}$ constant Voltage). Selected bands were cut out and the DNA Origami-AuNPs complexes were extracted from the gel with Freeze'N'Squeeze columns (Bio-Rad Laboratories Inc) at $4^{0} \mathrm{C}$.

\subsection{TEM Imaging}

Transmission electron micrographs were obtained with a JEM-2100 (HR) TEM. The sample was negatively stained. A $3 \mu \mathrm{l}$ sample solution was deposited onto the carbon-coated side of the TEM grid and allowed to adsorb for about 5 minutes. The grid sample-side was first immersed into a $\%$ uranyl acetate (Structure Probe, Inc) stainsolution droplet and incubated for 40 seconds. Excess liquid was dabbed off with the edge of filter paper, and the grid allowed to dry completely. Images were taken at different accelerating voltages.

The cross-like structure (Amoako et al., 2015) was used to organize AuNP helices formed on 100 nm DNA origami nanotube (Amoako et al., 2013) template. The attachment of AuNPs to DNA origami has been well explained in (Amoako, Zhou, Eghan, \& Sackey, 2018). To connect the cross-like structure to the nanoheliced DNA origami nanotubes, four attachment sites were designed at the four extreme ends of the DNA origami cross-like structure. The sequences labeled D3 to G4 in Table 1 were extended at the 3' end with an 8 base sequence ATGCATGC to get respectively sequences D1 to G2 in Table 1 . These 8 base extensions were to give sufficient flexibility to the DNA strands so that the structure will not be strained. This is a significant aspect of structural design in that it makes it easier for biotinylated strands from two structures to have easy access to the binding streptavidin. D1 to G2 were then biotinylated at the 3' end. One of the designed sites is shown in Figure 1 (shown with violet colors). To fold this DNA origami cross-like structure, all the caDNAno generated DNA sequences for the DNA origami cross-like structure were used except those at the connection sites which were replaced by the sequences D1, D2, E1, E2, F1, F2, G1, and G2 as shown in Table 1. The sequences from the designed connection sites were extended with biotin.

The AuNP helices were formed using a DNA origami nanotube as the template. To connect the DNA origami AuNP helices to the cross-like structure, one connection site was designed at one side of the nanotube template. Two sequences $\mathrm{K} 1$ and $\mathrm{K} 5$ shown in Table 1 were biotinylated at the designed connection site at the end of the DNA origami nanotube.

Table 1. Staple DNA sequences with the extended sticky ends. D1 to G2 are the extended and biotinylated sequences derived from the D3 to G4 respectively from the designed connection sites on the DNA origami crosslike structure. $\mathrm{K} 1$ and $\mathrm{K} 2$ are the sequences that were biotinylated at the end of the DNA origami nanotube

\begin{tabular}{lll}
\hline D1 & GCGAAGGCGATTCACAATTTTCCTGTGTGAAATTGCGTATGCATGC & 46 \\
D2 & AACTAACATTACGTTATTAGACTTTACAAACAATTCGACAATGCATGC & 48 \\
E1 & GTGACGACCAGTACATTGGCAGATTCACAAAAATGCATGC & 40 \\
E2 & TTAATCAAAGGGCGAAAAACCGTCTAATGCATGC & 34 \\
F1 & AATTAATCGCGCTAAAACATCAGCAGCAAATGCATGC & 37 \\
F2 & CCGTAGTTACAAACCTGAGCTTAATGCGCGAACTGATATGCATGC & 45 \\
G1 & AGGCCAGTCGGGAAACCTGTCATGCATGC & 29 \\
G2 & AGCTCAGCTGCACCTTCACCGCATGCATGC & 30 \\
D3 & GCGAAGGCGATTCACAATTTTCTGTGTGAATTGCGT & 38 \\
D4 & AACTAACATTACGTTATTAGACTTTACAAACAATTCGACA & 40 \\
E3 & GTGACGACCAGTACATTGGCAGATTCACAAAA & 32 \\
E4 & TTAATCAAAGGGCGAAAAACCGTCTA & 26 \\
F3 & AATTAATCGCGCTAAAACATCAGCAGCAA & 29 \\
F4 & CCGTAGTTACAAACCTGAGCTTAATGCGCGAACTGAT & 37 \\
G3 & AGGCCAGTCGGGAAACCTGTC & 21 \\
G4 & AGCTCAGCTGCACCTTCACCGC & 22 \\
K1 & ATGCATGCGTGGCGAGAAAGGAA & 23 \\
K2 & ATGCATGCTTAGTCTTTAA & 19 \\
\hline
\end{tabular}




\section{Results and Discussion}

After annealing and purification of the folded cross-like structures, a $20 \mu \mathrm{M} 20 \mu \mathrm{l}$ solution of streptavidin was added to a purified $20 \mu \mathrm{l}$ solution of the origami cross-like structures and the mixture was incubated overnight at $4^{\circ} \mathrm{C}$. After incubation, unbound streptavidin was removed using spin column filtration. The resulting solution was again washed with EDTA. After washing, the solution was mixed with purified $20 \mu \mathrm{l}$ of the nanoheliced DNA origami nanotube solution and the mixture incubated for two days at $4{ }^{\circ} \mathrm{C}$. Since streptavidin has four binding sites to biotin, one streptavidin molecule will bind together the DNA origami cross-like structure and the nanoheliced DNA origami nanotube at one end of the DNA origami cross-like structure. The same process occurred at the remaining three connection sites on the DNA origami cross-like structure.

When streptavidin was added to the annealed DNA cross-like origami solution, two of its binding sites bonded with the two biotin molecules attached to the DNA origami template at one side. The same process also happens at the remaining three connection sites of the DNA origami template. In all the situations, two binding sites on the streptavidin are left free to bind to the biotin attached to the new DNA origami structure. We characterized the structures with TEM.

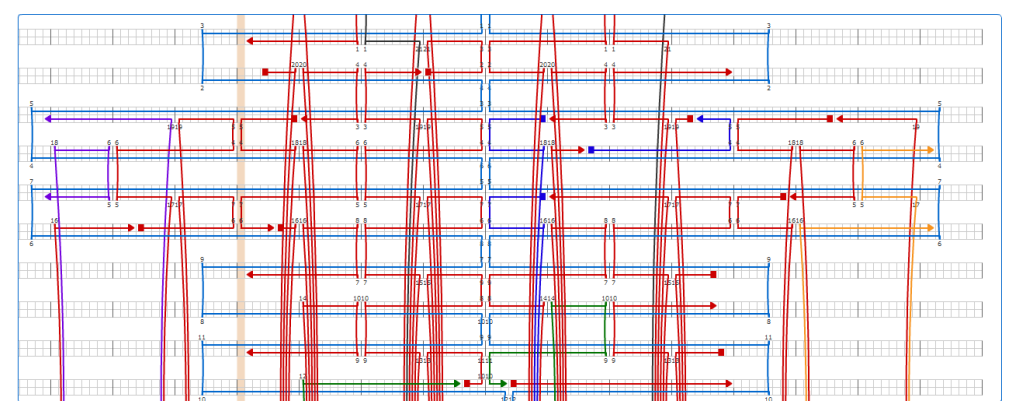

Figure 1. caDNAno interface of the Slice panel showing a designed connection site (shown with violet color)

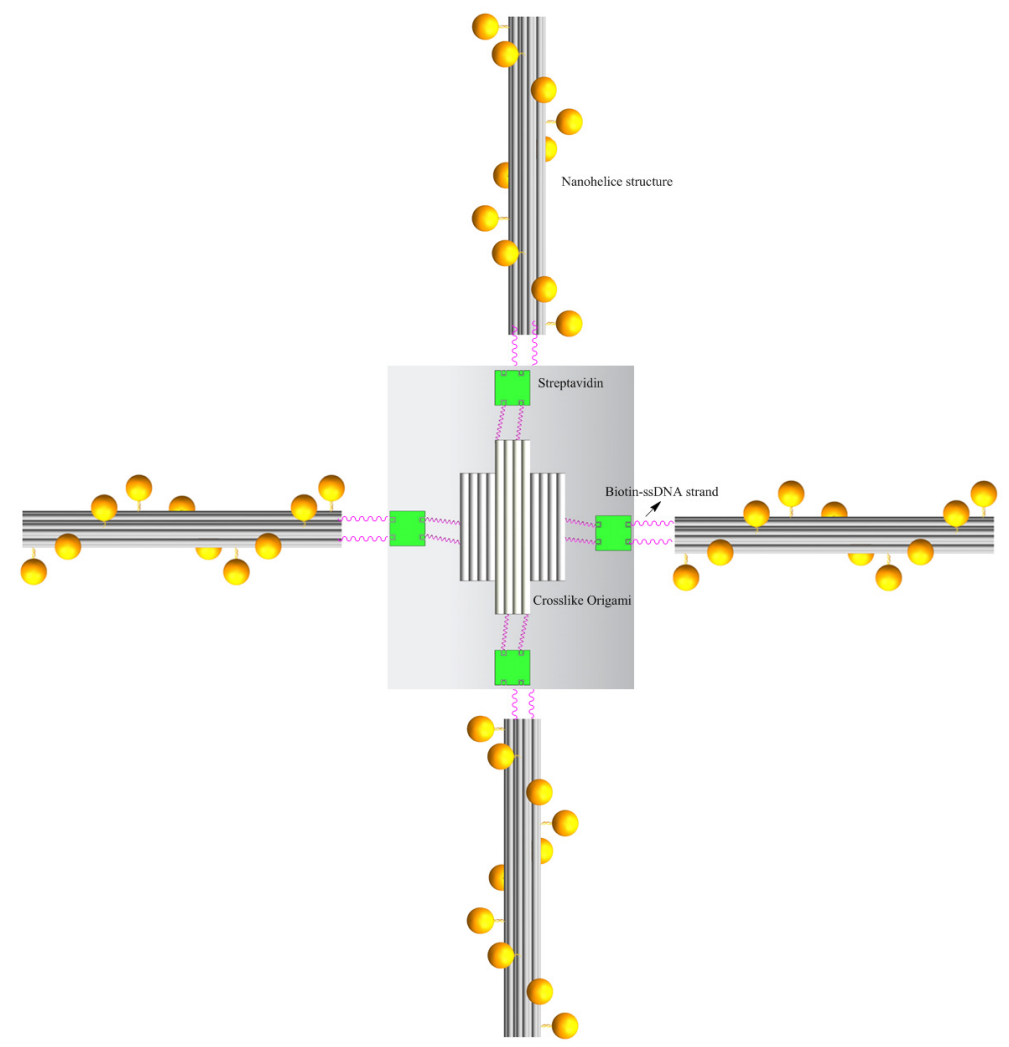

Figure 2. Schematic of the biotin-streptavidin connected 3D cross-like structure and the four DNA origami nanotube templated AuNPs helices 
The DNA origami AuNP helices were formed following the same folding and attachment processes already discussed in (Amoako et al., 2013). Figure 2 shows the schematic depiction of the cross-like structure connecting four templated AuNPs helices. TEM images were taking of these prepared samples and are shown in Figure 3.

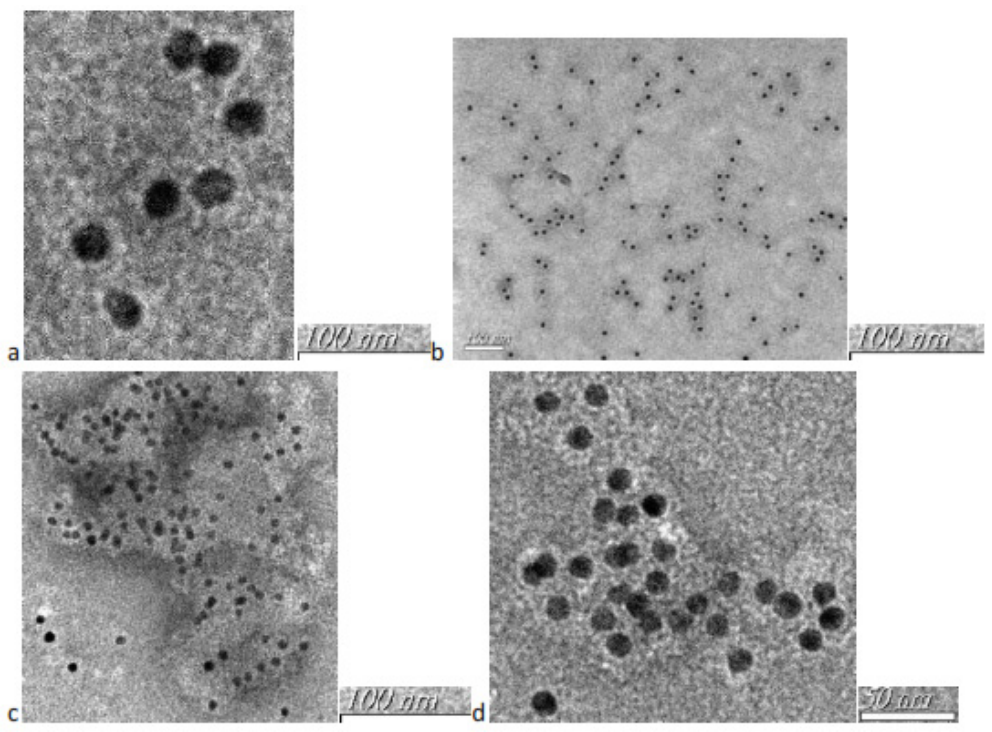

Figure 3. TEM images of the nanohelices and their connections by cross-like DNA origami object biotinylated at 4 different sites. (a) Au nanohelix formed by seven AuNPs. (b-d) Different scales of the images of the four nanohelices connected by the cross-like structure

Figure 3a shows the nanohelix formed by the AuNPs. It shows missing AuNPs so the helix was formed by seven particles instead of the ten used (ten attachment sites were designed for each). Figure 3b-d show the Au nanohelices connected by the cross-like DNA origami structure with the sequences at four sites biotinylated. The mixture showed a great tendency to form large aggregates. The connections are clearly seen in the images but with a lot of distortions. It is believed that the distortions were due to the preparations on the TEM grids and the large dimensions of the composite structure. But the formation of the structure (network) has clearly been demonstrated.

\section{Conclusion}

In this work, connecting DNA origami structures into a network has been demonstrated. Use was made of the strong specific affinity interaction between biotin and streptavidin to connect the DNA origami templated structures. The DNA origami structures were functionalized with gold nanoparticles. There were severe distortions with the network formed. These distortions could be attributed to the deposition of the DNA origami structures on the TEM grid.

\section{Acknowledgment}

I acknowledge the contributions of Ye Rian in the performance of the experiments.

\section{Conflict of interests}

The authors declare that there is no conflict of interests regarding the publication of this paper.

\section{References}

Acuna, G. P., Bucher, M., Stein, I. N., Steinhauer, C., Kuzyk, A., Holzmeister, P., Schreiber, R., Moroz, A., Stefani, F. D., Liedl, T., Simmel, F. C., \& Tinnefeld, P. (2012). Distance Dependence of Single Fluorophore Quenching by Gold Nanoparticles Studied on DNA Origami. ACS Nano, 6, 3189-3195.

Amoako, G., Ye, R., Zhuang, L., Yang, X., Shen, Z., \& Zhou, M. (2013). DNA origami site-specific arrangement of gold nanoparticles. Nano, 8, 1350064.

Amoako, G., Zhou, M., Eghan, M. J., \& Sackey, S. S. (2018). DNA Origami as a Tool to Design Asymmetric Gold Nanostructures. Journal of Materials Science Research, 7(1), 1-8.

Amoako, G., Zhou, M., Ye, R. A., Zhuang, L., Yang, X., \& Shen, Z. (2013). 3D DNA origami designed with caDNAno. Chinese Science Bulleting, 58, 3018-3022. 
Amoako, G., Zhou, M., Ye, R., Mensah-Amoah, P., Twum, A., \& Sam, F. (2015). Connecting DNA origami structures using the biotin-streptavidin specific binding. African Journal of Biotechnology, 14(28), 2258-2264.

Bain, C. D., \& Whitesides, G. M. (1989). Modeling organic surfaces with self-assembled monolayers. Angewandte Chemie International Edition in English, 28, 506-512.

Boltasseva, A., \& Shalaev, V. M. (2008). Fabrication of optical negative-index metamaterials: recent advances and outlook. Metamaterials, 2, 1-17.

Bruchez, M., Moronne, M., Gin, P., Weiss, S., \& Alivisatos, A. P. (1998). Semiconductor Nanocrystals as Fluorescent Biological Labels. Science, 281, 2013-2016.

Cai, W., \& Shalaev, V. (2010). Optical Metamaterials Fundamentals and Applications. Springer.

Castro, C. E., Kilchherr, F., Kim, D. N., Shiao, E. L., Wauer, T., Wortmann, P., Bathe, M., \& Dietz, H. (2011). A primer to scaffolded DNA origami Nature Methods, 8, 221-229.

Connolly, S., \& Fitzmaurice, D. (1999). Programmed Assembly of Gold Nanocrystals in Aqueous Solution. Advanced Materials, 11, 1202-1205.

Ding, B., Deng, Z., Yan, H., Cabrini, S., Zuckermann, R. N., \& Bokor, J. (2010). Gold nanoparticle self-similar chain structure organized by DNA Origami. Journal of American Chemical Society, 132, 3248-3249.

Douglas, S. M., Dietz, H., Liedl, T., Hoegberg, B., Graf, F., \& Shih, W. M. (2009). Self-Assembly of DNA into nanoscale three-dimensional shapes. Nature, 459, 414-418.

Douglas, S. M., Marblestone, A. H., Teerapittayanon, S., Vazquez, A., Church, G. M., \& Shih, W. M. (2009). Rapid prototyping of 3D DNA-origami shapes with caDNAno. Nucleic Acids Research, 37, 5001-5006.

Dubois, L. H., \& Nuzzo, R. G. (1992). Synthesis, Structure, and Properties of Model Organic Surfaces. Annual Review of Physical Chemistry, 43, 437-463.

Hermanson, G. T. (1996). Bioconjugate techniques. Academic Press.

Herne, T. M., \& Tarlov, M. J. (1997). Characterization of DNA probes immobilized on gold surfaces. Journal of American Chemical Society, 119, 3401-3402.

Jin, R., Wu, G., Li, Z., Mirkin, C. A., \& Schatz, G. C. (2003). What Controls the Melting Properties of DNALinked Gold Nanoparticle Assemblies? Journal of America Chemical Society, 125, 1643-1654.

Kuzyk, A., Schreiber, R., Fan, Z., Pardatscher, G., Roller E. M., Hoegele, A., Simmel, F. C., Govorov, A. O., \& Liedl, T. (2012). DNA-based self-assembly of chiral plasmonic nanostructures with tailored optical response. Nature, 483, 311-314.

Maier, S. A., Brongersma, M. L., Kik, P. G., Meltzer, S., Requicha, A. A. G., \& Atwater, H. A. (2001). Plasmonics - A Route to Nanoscale Optical Devices. Advanced Materials, 13, 1501-1505.

Mirkin, C. A., Letsinger, R. L., Mucic, R. C., \& Storhoff, J. J. (1996). A DNA-Based Method for Rationally Assembling Nanoparticles into Macroscopic Materials. Nature, 382, 607-609.

Moriarty, P. (2001). Nanostructured materials. Reports on Progress in Physics, 64, 297-381.

Mucic, R. C., Storhoff, J. J., Mirkin, C. A., \& Letsinger, R. L. (1998). DNA Directed Synthesis of Binary Nanoparticle Network Materials. Journal of America Chemical Society, 120, 12674-12675.

Nuzzo, R. G., \& Allara, D. L. (1983). Adsorption of Bifunctional Organic Disulfides on Gold Surfaces. Journal of American Chemical Society, 105, 4481-4483.

Peyrard, M. (2004). Nonlinear dynamics and statistical physics of DNA. Nonlinearity, 17, R1-R40.

Pilo-Pais, M., Goldberg, S., Samano, E., LaBean, T. H., \& Finkelstein, G. (2011). Connecting the Nanodots: Programmable Nanofabrication of Fused Metal Shapes on DNA Templates. Nano Letters, 11, 3489-3492.

Ramesh, K. T. (2009). Nanomaterials: Mechanics and Mechanisms. Springer.

Ratner, M., \& Ratner, D. (2003). Nanotechnology: A Gentle Introduction to the Next Big Idea. Prentice Hall PTR.

Rosei, F. (2004). Nanostructured surfaces: Challenges and frontiers in nanotechnology. Journal of PhysicsCondensed Matter, 16, S1373-S1436.

Rothemund, P. (2006). Folding DNA to create nanoscale shapes and patterns. Nature, 440, 297-302.

Seeman, N. C. (1982). Nucleic acid junctions and lattices. Journal of Theoretical Biology, 99, 237-247. 
Seeman, N. C. (2003). DNA in a material world. Nature, 421, 427-431.

Shen, X., Song, C., Wang, J., Shi, D., Wang, Z., Liu, N., \& Ding, B. (2012). Rolling up gold nanoparticle-dressed DNA origami into threedimensional plasmonic Chiral nanostructures. Journal of American Chemical Society, 134, 146-149.

Shenton, W., Davis, S. A., \& Mann, S. (1999). Directed Self-Assembly of Nanoparticles into Macroscopic Materials Using Antibody-Antigen Recognition. Advanced Materials, 11, 449-452.

Storhoff, J. J., \& Mirkin, C. A. (1999). Programmed materials synthesis with DNA. Chemical Reviews, 99, 18491862.

Storhoff, J. J., Elghanian, R., Mucic, R. C., Mirkin, C. A., \& Letsinger, R. L. (1998). One-Pot Colorimetric Differentiation of Polynucleotides with Single Base Imperfections Using Gold Nanoparticle Probes. Journal of America Chemical Society, 120, 1959-1964.

Sun, Y., \& Kiang, C.-H. (2005). Handbook of Nanostructured Biomaterials and Their Applications in Nanobiotechnology, 2(ed Nalwa), 224-246.

Tanton, T. A. (2002). Preparation of Gold Nanoparticle-DNA Conjugates. Current Protocols in Nucleic Acid Chemistry, 12.12.11-12.12.12.

Whaley, S. R., English, D. S., Hu, E. L., Barbara, P. F., \& Belcher, A. M. (2000). Selection of Peptides with Semiconductor Binding Specificity for Directed Nanocrystal Assembly. Nature, 405, 665-668.

Yang, M., Yau, H. C. M., \& Chan, H. L. (1998). Adsorption Kinetics and Ligand-Binding Properties of ThiolModified Double-Stranded DNA on a Gold Surface. Langmuir, 14, 6121-6129.

Zhao, Z., Jacovetty, E. L., Liu, Y., \& Yan, H. (2011). Encapsulation of Gold Nanoparticles in a DNA Origami Cage. Angewandte Chemie International Edition, 50, 2041-2044.

\section{Copyrights}

Copyright for this article is retained by the author(s), with first publication rights granted to the journal.

This is an open-access article distributed under the terms and conditions of the Creative Commons Attribution license (http://creativecommons.org/licenses/by/4.0/). 